\title{
Improvement in Mineral Digestibility and Whole Body Composition of Catla catla Fingerlings Fed Phytase Supplemented MOSM Based Diet
}

\author{
Muhammad Mudassar Shahzad ${ }^{1,2}$, Syed Makhdoom Hussain',*, Farhat Jabeen², \\ Abdullah Ijaz Hussain ${ }^{3}$, Arshad Javid ${ }^{4}$, Muhammad Asrar ${ }^{2}$ and \\ Muhammad Zubair-ul-Hassan Arsalan² \\ ${ }^{1}$ Department of Zoology, Division of Science and Technology, University of Education \\ Township Campus Lahore \\ ${ }^{2}$ Fish Nutrition Lab, Department of Zoology, Government Collage University, Faisalabad \\ ${ }^{3}$ Department of Chemistry, Government Collage University, Faisalabad \\ ${ }^{4}$ Department of Wildlife and Ecology, University of Veterinary and Animal Sciences, \\ Lahore
}

\begin{abstract}
A B S T RA C T
Moringa oleifera seed meal (MOSM) was used as test ingredient to formulate six test diets and were supplemented with graded levels $\left(0,300,600,900,1200\right.$ and $\left.1500 \mathrm{FTU} \mathrm{kg}^{-1}\right)$ of phytase. The fingerlings were fed at the rate of $4 \%$ of live fish body weight twice a day and faeces were collected from each tank. Present research work was carried out to determine the effect of phytase supplementation on mineral digestibility and carcass composition of Catla catla fingerlings fed MOSM based diets. Phytate in plant by-products decreases the bioavailability of minerals and deposition of nutrients in fish body, resulting in poor fish growth. Results demonstrated that phytase supplementation showed significant $(p<0.05)$ improvement in $\mathrm{ADC} \%$ of minerals and carcass composition of fish. Maximum digestibility of minerals and improved carcass composition of $C$. catla fingerlings was noted at $900 \mathrm{FTU} \mathrm{kg}^{-1}$ level of phytase supplemented MOSM based test diet. It was further noted that phytase supplementation decreases the discharge of minerals through faeces resulting in reduced aquatic pollution. It was concluded that phytase supplementation at $900 \mathrm{FTU} \mathrm{kg}^{-1}$ level was helpful to develop an eco-friendly and cost effective fish feed by using MOSM based diet.
\end{abstract}

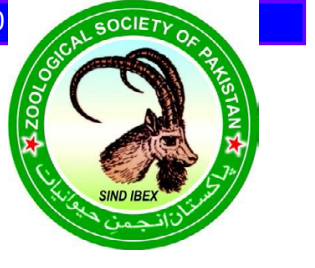
CrossMark

\begin{tabular}{|c|}
\hline Article Information \\
\hline Received 04 December 2016 \\
\hline Revised 27 August 2017 \\
\hline Accepted 24 April 2018 \\
\hline Available online 17 August 2018 \\
\hline Authors' Contribution \\
\hline $\begin{array}{l}\text { MMS conducted the research and } \\
\text { wrote the manuscript. SMH planned } \\
\text { and supervised the research. FJ } \\
\text { provided the research facilities. } \\
\text { MIH helped in chemical analysis } \\
\text { of minerals. AJ helped in statistical } \\
\text { analysis and interpretation of the } \\
\text { results. MA helped in manuscript } \\
\text { preparation. MZHA helped in } \\
\text { conducting feeding trial and feces } \\
\text { collection. }\end{array}$ \\
\hline Key words \\
\hline $\begin{array}{l}\text { MOSM, Catla catla, Mineral } \\
\text { digestibility, Phytase, Carcass } \\
\text { composition. }\end{array}$ \\
\hline
\end{tabular}

Article Information

Revised 27 August 2017 Accepted 24 April 2018

vailable online 17 August 2018

and supervised the research. FJ

provided the research facilities.

of minerals. AJ helped in statistical

analysis and interpretation of the

results. MA helped in manuscript

collection.

composition.

\section{INTRODUCTION}

$M$ oringa oleifera seed meal (MOSM) contains essential minerals such as $\mathrm{Ca}, \mathrm{K}, \mathrm{Fe}, \mathrm{Mg}, \mathrm{Cu}$ and $\mathrm{Zn}$ etc. (Anjorin et al., 2010). It is also a good source of protein, vitamins and essential amino acids EAA (Makkar and Becker, 1996). These EAA are comparatively less in other plant ingredients such as soybean meal (Ferreira et al., 2008). The seed protein contents are higher (33$38 \%$ ) than those reported for important grain legumes and soybean varieties (Ferreira et al., 2008). Analysis of the proximate composition of moringa seeds have also shown the higher levels of crude fat, fiber, protein, carbohydrates and minerals (Abdulkarim et al., 2005).

Over population has resulted in increasing demand for food and nutritional quality in under developed countries throughout the world (Abdulkadir et al., 2016). Now a day

\footnotetext{
Corresponding author: drmakhdoom90@gmail.com 0030-9923/2018/0005-1909 \$ 9.00/0

Copyright 2018 Zoological Society of Pakistan
}

in food producing sectors, aquaculture is one of the rapidly flourishing industries in recent decades because of the need for high quality fish protein to meet human's nutritional requirements (Tacon and Metian, 2013). Nearly 40\% of shellfish and fish that is being eaten by human are reared in aquaculture sector (Chabi et al., 2015). As fish is the most important source of protein, it also needs a high amount of protein in its own diet. Aquaculture feed industry usually depends on the use of fishmeal (FM) as it is major source of protein and contains important vitamins, minerals and nutrients (Dawood et al., 2015). Fish feed mainly accounts for $60 \%$ of total cost of fish culture system (Essa et al., 2004). Whereas, unstable supply, higher demand and increasing cost of the fish meal made it compulsory to search for unconventional and low cost sources of protein (Rana et al., 2009; FAO, 2014). The best alternatives of protein sources are plant by-products because of their low cost and easy availability throughout the year. Use of plant ingredients as protein sources singly or in mixture of two or more plant by-products appears economically more useful (Enterria et al., 2011). A number of researchers have 
found positive effects on mineral digestibility and carcass compositions when different fish species were fed on plant by-products based diets (Chu et al., 2015; Hussain et al., 2015a, b; Liu et al., 2015; Muin et al., 2015). Plant byproducts contain several anti-nutritional factors such as phytate, Tannins, trypsin inhibitors, saponins, oxalates and cyanide contents. Majority of these anti-nutritional factors can be easily removed by heat, soaking or by extraction methods (Liener, 1994), whereas phytic acid or phytate cannot be removed by these methods (Plaipetch and Yakupitiyage, 2014). Phytate is chemically known as myo-inositol hexaphosphate that is chelated form of phosphorus. Availability of minerals especially cation minerals, protein and lipids are adversely affected by higher concentration of phytate or phytic acid in plant byproducts or oilseeds meals based diets (Cao et al., 2007). It is estimated that $60-80 \%$ of total $\mathrm{P}$ is present in chelated form that is known as phytic acid in plant by-products based diets (Lei et al., 2013). This chelated phosphorous cannot be utilized by mono-gastric and a-gastric fishes, resulting in higher mineral discharge into water media causing aquatic pollution (NRC, 1993). Moreover, phytate prevents absorption of divalent minerals such as $\mathrm{Fe}, \mathrm{Ca}$, $\mathrm{Mg}, \mathrm{Zn}, \mathrm{Mn}$ and $\mathrm{Cu}$ in fish digestive system. It is also capable of binding with proteins and starch (Jondreville et al., 2005; Noureddini and Dang, 2009). Phytate complex only can be broken down by some enzymatic reactions because it is a stable compound (Vielma et al., 2000).

Phytase is chemically known as myo-inositol hexakis-phosphate phospho-hydrolase, belongs to class III hydrolases. Fermentation of genetically modified microorganisms, phytase can be produced at low cost (Yao and Fan, 2000). Phytase supplementation in plant byproducts is being extensively used to get free $\mathrm{P}$ from phytic acid complexes (Lim and Lee, 2009). Supplemental dietary phytase is an effective method to improve the availability of minerals and carcass composition of fish. It also decreases water pollution by proper digestion and absorption of $\mathrm{P}$ in fish body (Liu et al., 2013; Hussain et al., 2015a). Use of phytase can efficiently decrease the adverse effects of phytic acid that is present in plant by-products based diets (Hussain et al., 2016; 2017). Phytase deficiency may cause three main problems due to less availability of minerals i.e. 1) water pollution caused by minerals discharge through fish feces 2) need of extra dietary P supplementation 3) as well as the depletion of rock P deposits (Lei et al., 2013). Supplementation of dietary phytase in plant byproducts based diet significantly improves the fish carcass composition (Hung et al., 2015). It also improves protein absorption and increase digestibility of minerals such as $\mathrm{P}, \mathrm{N}, \mathrm{Mg}, \mathrm{Ca}, \mathrm{Cu}, \mathrm{Zn}$, and $\mathrm{Fe}$ in oilseed meal based diets (Christopher et al., 2011; Hussain et al., 2016).
C. catla commonly known as Thaila, is being cultured in Pakistan with other carps (Aslam et al., 2016). The reported production of $C$. catla has been increased during the first decade of $21^{\text {st }}$ century and in 2012 it was about 2.8 million ton/annum (FAO, 2015). Research is needed to enhance the fish production as well as to overcome the problems of costly fish meal and water pollution caused by excretion of chelated phosphorus through fish feces. Therefore, the purpose of this study was to examine the effects of phytase supplementation on mineral availability and carcass composition of $C$. catla fingerlings fed MOSM based test diets.

\section{MATERIALS AND METHODS}

The present research work is performed to investigate the impact of phytase supplementation on mineral digestibility and carcass composition of $C$. catla fingerlings fed MOSM based test diets. The experiment was conducted in Fish Nutrition Laboratory, Department of Zoology, Government College University Faisalabad, Pakistan.

\section{Fish and experimental conditions}

C. catla fingerlings were procured from the Government Fish Seed Hatchery, Satiana Road, Faisalabad. Prior to experiment, the fingerlings were acclimatized to the experimental conditions for fourteen days. Fingerlings were stocked in specially designed V-shaped water tanks having $70 \mathrm{~L}$ water capacity and fed once daily on basal $\operatorname{diet}$ (Allan and Rowland, 1992). Water quality parameters such as temperature, $\mathrm{pH}$ and dissolved oxygen (DO) were monitored on daily basis. Air pump was used to supply air by capillary system through-out the experimental period. Before the start of feeding trial fingerlings were treated for 1 to 2 minutes with $0.5 \%$ saline solution to remove the pathogens if present (Rowland and Ingram, 1991).

\section{Experimental design}

Moringa oleifera seed meal (MOSM) was used as test ingredient to formulate six test diets and supplemented with graded levels $(0,300,600,900,1200$ and 1500 FTU $\mathrm{kg}^{-1}$ ) of phytase enzyme. One control and five phytase supplemented MOSM based test diets were fed to six fish groups stocked in water tanks. Triplicate tanks were used for each treatment and in each replicate 15 fingerlings were stocked. Duration of the experiment was 90-days. Each MOSM based diet supplemented with phytase was compared with other as well as control diet to determine $\mathrm{ADC} \%$ of minerals and carcass composition by using completely randomized design (CRD). 
Table I.- Ingredients composition (\%) of control and test diets (dry matter basis).

\begin{tabular}{lcccccc}
\hline Ingredients & Test diet-I (Control) & Test diet-II & Test diet-III & Test diet-IV & Test diet-V & Test diet-VI \\
\hline Phytase level (FTU kg-1) & 0 & 300 & 600 & 900 & 1200 & 1500 \\
MOSM & 35 & 35 & 35 & 35 & 35 & 35 \\
Wheat flour* & 17 & 17 & 17 & 17 & 17 & 17 \\
Fish meal & 15 & 15 & 15 & 15 & 15 & 15 \\
Soybean meal & 15 & 15 & 15 & 15 & 15 & 15 \\
Rice polish & 8 & 8 & 8 & 8 & 6 & 8 \\
Fish oil & 6 & 6 & 6 & 1.0 & 1.0 & 6 \\
Vitamin Premix $* *$ & 1.0 & 1.0 & 1.0 & 1.0 & 1.0 & 1.0 \\
Chromic oxide & 1.0 & 1.0 & 1.0 & 1.0 & 1.0 & 1.0 \\
Ascorbic acid & 1.0 & 1.0 & 1.0 & 1.0 & 1.0 & 1.0 \\
Mineral mixture $* * *$ & 1.0 & 1.0 & 1.0 & 1.0 & \\
\hline
\end{tabular}

MOSM= M. oleifera seed meal. *Phytase enzyme was used at the expense of wheat flour. **vitamin premix $/ \mathrm{kg}$ : Vitamin $\mathrm{D}_{3}, 3,000,000 \mathrm{IU}$; Vitamin A, 15,000,000 IU; Vitamin E, $30000 \mathrm{IU}$; Vitamin B, $3000 \mathrm{mg}$; Vitamin B , $4000 \mathrm{mg}$; Vitamin B, 40 mg; Vitamin B, 7000 mg; Vitamin C, 15,000 mg; Vitamin $\mathrm{K}_{3}, 8000 \mathrm{mg}$; Folic acid, $1500 \mathrm{mg}$; Calcium pantothenate, 12,000 mg; Nicotinic acid, $60,000 \mathrm{mg}$. ***mineral premix/kg: Mn(Manganese), 2000 mg; Ca (Calcium), 155 gm; Zn (Zinc), 3000 mg; Cu (Copper), 600 mg; Co (Cobalt), 40 mg; I (Iodine), 40 mg; P (Phosphorous), 135 g; Fe (Iron), 1000 mg; Mg (Magnesium), 55 gm; Se (Selenium), 3 mg; Na (Sodium), 45 gm.

Table II.- Chemical composition (\%) of feed ingredients (dry matter basis).

\begin{tabular}{lccccc}
\hline Ingredients & MOSM & Fish meal & Rice polish & Wheat flour & Corn gluten meal (60\%) \\
\hline Dry matter (\%) & 93.74 & 91.67 & 94.06 & 92.4 & 92.34 \\
Crude protein (\%) & 34.41 & 48.17 & 12.38 & 10.15 & 59.51 \\
Crude fat (\%) & 4.28 & 7.12 & 13.46 & 2.3 & 4.58 \\
Crude fiber (\%) & 2.03 & 1.12 & 12.74 & 2.67 & 1.23 \\
Ash (\%) & 9.63 & 24.66 & 10.17 & 2.06 & 1.36 \\
Gross energy (kcal/g) & 4.17 & 2.65 & 3.18 & 2.95 & 4.35 \\
Carbohydrates & 45.48 & 16.28 & 48.07 & 79.87 & 28.97 \\
\hline
\end{tabular}

Processing of Moringa seeds and formation of feed pellets

Feed ingredients (Table I) were obtained from local market of Faisalabad. M. oleifera seeds were air-dried and de-fatted by press method (Weiss, 1971; Salem and Makkar, 2009). Ingredients were finely grinded to pass through $0.3 \mathrm{~mm}$ sieve size. Feed ingredients were analyzed for chemical composition (Table II) by following standard methods of AOAC (2000). $\mathrm{Cr}_{2} \mathrm{O}_{3}$ was used as inert marker at the rate of $1 \%$ in all the test diets. All feed ingredients were thoroughly mixed in a feed mixer for about 5-10 min whereas fish oil was also added during this process. Feed ingredients were blended slowly into the mixer after adding $10-15 \%$ of tap water, resulting in suitably textured dough and were processed through pelleting machine to formulate pellets (Lovell, 1989). One control and five phytase supplemented test diets were prepared using MOSM based diet by spraying graded levels $(0,300,600$, 900, 1200 and $1500 \mathrm{FTU} \mathrm{kg}^{-1}$ ) of phytase. The required concentrations of phytase enzyme were prepared in $25 \mathrm{~mL}$ distilled water and sprayed on each test diet (Robinson et al., 2002). Control diet (0 FTU kg-1 level) was sprayed with a similar amount of water to maintain the equivalent amount of moisture. All diets were dried and stored at $4^{\circ} \mathrm{C}$ until use.

\section{Feeding protocol and sample collection}

C. catla fingerlings were fed at the rate of $4 \%$ of live wet fish body weight on their prescribed diet twice daily. After the feeding session of two hours, the uneaten diet was drained out from each tank by opening the valves of the tanks. The tanks were washed completely to remove the feed particles and refilled with tap water. Feces were collected by the opening fecal collecting tube of each replicated tank. Fecal material was collected carefully to avoid the breakage of faeces to minimize the leaching of minerals in water. Faeces were dried in oven at $65^{\circ} \mathrm{C}$ and stored for further chemical analysis. 
Table III.- Analyzed mineral composition (\%) of MOSM based test and control diets.

\begin{tabular}{|c|c|c|c|c|c|c|}
\hline Minerals & $\begin{array}{c}\text { Test diet -I } \\
\text { (control diet) }\end{array}$ & Test diet -II & Test diet -III & Test diet -IV & Test diet -V & Test diet-VI \\
\hline $\begin{array}{l}\text { Phytase levels } \\
(\text { FTU kg-1) }\end{array}$ & 0 & 300 & 600 & 900 & 1200 & 1500 \\
\hline $\mathrm{Ca}$ & $0.064 \pm 0.0018$ & $0.064 \pm 0.0012$ & $0.066 \pm 0.00057$ & $0.064 \pm 0.0038$ & $0.064 \pm 0.0034$ & $0.063 \pm 0.0018$ \\
\hline $\mathrm{Na}$ & $0.0074 \pm 0.0003$ & $0.0074 \pm 0.0005$ & $0.0076 \pm 0.0005$ & $0.0073 \pm 0.0006$ & $0.0075 \pm 0.0005$ & $0.0075 \pm 0.0008$ \\
\hline $\mathrm{K}$ & $1.33 \pm 0.05$ & $1.34 \pm 0.06$ & $1.35 \pm 0.04$ & $1.33 \pm 0.06$ & $1.34 \pm 0.06$ & $1.35 \pm 0.07$ \\
\hline $\mathrm{Fe}$ & $0.053 \pm 0.001$ & $0.052 \pm 0.002$ & $0.055 \pm 0.003$ & $0.053 \pm 0.002$ & $0.055 \pm 0.003$ & $0.054 \pm 0.005$ \\
\hline $\mathrm{Cu}$ & $0.0033 \pm 0.00003$ & $0.0033 \pm 0.0001$ & $0.0033 \pm 0.0001$ & $0.0034 \pm 0.00003$ & $0.0033 \pm 0.00004$ & $0.0033 \pm 0.0001$ \\
\hline $\mathrm{Zn}$ & $0.047 \pm 0.0005$ & $0.047 \pm 0.001$ & $0.047 \pm 0.001$ & $0.048 \pm 0.0004$ & $0.047 \pm 0.001$ & $0.047 \pm 0.0005$ \\
\hline $\mathrm{Mn}$ & $0.021 \pm 0.001$ & $0.021 \pm 0.001$ & $0.021 \pm 0.001$ & $0.021 \pm 0.001$ & $0.021 \pm 0.001$ & $0.021 \pm 0.001$ \\
\hline $\mathrm{P}$ & $2.11 \pm 0.02$ & $2.1 \pm 0.02$ & $2.1 \pm 0.04$ & $2.1 \pm 0.03$ & $2.12 \pm 0.02$ & $2.11 \pm 0.03$ \\
\hline $\mathrm{Mg}$ & $0.0088 \pm 0.0003$ & $0.0090 \pm 0.0002$ & $0.0089 \pm 0.0006$ & $0.0090 \pm 0.0003$ & $0.0089 \pm 0.0004$ & $0.0090 \pm 0.0004$ \\
\hline $\mathrm{Al}$ & $0.00053 \pm 0.00001$ & $0.00054 \pm 0.00001$ & $0.00053 \pm 0.00001$ & $0.00053 \pm 0.000004$ & $0.00053 \pm 0.00001$ & $0.00054 \pm 0.00001$ \\
\hline $\mathrm{Cr}$ & $0.032 \pm 0.003$ & $0.031 \pm 0.004$ & $0.032 \pm 0.004$ & $0.032 \pm 0.004$ & $0.031 \pm 0.01$ & $0.031 \pm 0.003$ \\
\hline $\mathrm{Sr}$ & $0.00009 \pm 0.000002$ & $0.00009 \pm 0.000003$ & $0.0001 \pm 0.000004$ & $0.00009 \pm 0.000003$ & $0.00009 \pm 0.000004$ & $0.00009 \pm 0.000005$ \\
\hline $\mathrm{Pb}$ & $0.0028 \pm 0.0001$ & $0.0028 \pm 0.0001$ & $0.0029 \pm 0.0001$ & $0.0029 \pm 0.0001$ & $0.0029 \pm 0.0001$ & $0.0029 \pm 0.0001$ \\
\hline $\mathrm{Ba}$ & $<0.0001$ & $<0.0001$ & $<0.0001$ & $<0.0001$ & $<0.0001$ & $<0.0001$ \\
\hline $\mathrm{Cd}$ & $<0.0001$ & $<0.0001$ & $<0.0001$ & $<0.0001$ & $<0.0001$ & $<0.0001$ \\
\hline $\mathrm{Co}$ & $<0.0001$ & $<0.0001$ & $<0.0001$ & $<0.0001$ & $<0.0001$ & $<0.0001$ \\
\hline Mo & $<0.0001$ & $<0.0001$ & $<0.0001$ & $<0.0001$ & $<0.0001$ & $<0.0001$ \\
\hline $\mathrm{Ni}$ & $<0.0001$ & $<0.0001$ & $<0.0001$ & $<0.0001$ & $<0.0001$ & $<0.0001$ \\
\hline
\end{tabular}

Data are means of three replicates.

\section{Chemical analysis of fish carcass}

After 90 days of feeding trial five fingerlings from each tank were selected randomly, sacrificed and dried at room temperature for carcass study. Moisture contents were calculated after oven-drying of homogenised samples at $105^{\circ} \mathrm{C}$ for $12 \mathrm{~h}$. Micro Kjeldahl Apparatus (InKjel M behr Labor Technik GmbH D-40599 Dusseldorf) was used to determine the crude protein $(\mathrm{CP})$ contents $(\mathrm{N} \times$ 6.25) whereas Soxhlet system (Soxhlet Extraction Heating Mantels, $250 \mathrm{ml} 53868601$ ) was used to analyse the crude fat (EE) by the help of ether extraction method. Crude fiber (CF) contents were calculated as loss on ignition of dried lipid-free residues after digestion with $1.25 \% \mathrm{H}_{2} \mathrm{SO}_{4}$ and $1.25 \% \mathrm{NaOH}$ whereas ash was determined by ignition at $650^{\circ} \mathrm{C}$ for $12 \mathrm{~h}$ in electric furnace (Naberthern B170) to constant weight. Total carbohydrates ( $\mathrm{N}$-free extract) were calculated by using standard formula i.e. Total carbohydrates $(\%)=100-(\mathrm{EE} \%+\mathrm{CP} \%+\mathrm{Ash} \%+\mathrm{CF}$ $\%)$. Oxygen bomb calorimeter was used to estimate the gross energy of samples.

\section{Estimation of minerals}

For estimation of minerals, $1 \mathrm{~g}$ of the sample (experimental feed and faeces) was weighed and put into an open mouth conical flask. Nitric acid was added $(20 \mathrm{ml})$ in the flask and placed it on hot plate. When the mixture began to boil, $10 \mathrm{ml}$ perchloric acid was added and again placed on hot plate and heated it until $1 \mathrm{ml}$ of the mixture was left behind. The flask was removed and diluted by adding distilled water until the volume $(50 \mathrm{ml})$ became crystal clear. This final solution was filtered by using filter paper to remove all particulate matter in the digestion solution prior to the analysis of minerals (AOAC, 2000). After appropriate dilution, mineral contents were estimated by using Atomic Absorption Spectrophotometer. The estimation of $\mathrm{Na}$ and $\mathrm{K}$ was done through flame photometer (Jenway PFP-7, UK).

\section{Determination of phosphorus $(P)$}

UV/VIS spectrophotometer at $720 \mathrm{~nm}$ absorbance was used to determine $\mathrm{P}$ contents in the experimental diets and feces. Ammonium molybdate $(2.5 \mathrm{~g})$ in $100 \mathrm{ml}$ of distilled $\mathrm{H}_{2} \mathrm{O}$ was used to prepare the ammonium molybdate reagent. Prepared solution was titrated against pure $\mathrm{H}_{2} \mathrm{SO}_{4}$ till it became clear. Sodium meta-bisulphite $(7.5 \mathrm{~g})$ was dissolved in $50 \mathrm{ml}$ of distilled $\mathrm{H}_{2} \mathrm{O}$ to prepare the amino-nepthol-sulphonic acid reagent and then added $0.125 \mathrm{~g}$ of amino nepthol in that prepared reagent. The reagent was titrated against $\mathrm{Na}_{2} \mathrm{SO}_{4}(20 \%)$ till 0 turbidity level. Standard $\mathrm{P}$ solution was prepared by dissolving $0.351 \mathrm{~g}$ potassium dihydrogen $\mathrm{P}$ in $100 \mathrm{ml}$ of distilled $\mathrm{H}_{2} \mathrm{O}$. Then the test tubes were marked as standard, test sample 
and blank and filled them with samples as marked for $\mathrm{P}$ analysis. Test tubes were shaken well and permitted to settle down for five minutes. Blue colour appeared in the solution containing P. At $720 \mathrm{~nm}$, the absorbance was noted on spectrophotometer after setting the zero with blank.

\section{Calculation of minerals $A D C$}

Apparent nutrient digestibility coefficients (ADC) (\%) of test diets were calculated by the standard formula (NRC, 1993):

ADC $(\%)=100-100 \times(\%$ marker in diet $\times \%$ minerals in faeces $/ \%$ marker in faeces $\times \%$ minerals in diet)

\section{Statistical analysis}

Finally, data of ADC\% of minerals (Ca, Na, K, Fe, $\mathrm{Cu}, \mathrm{Zn}, \mathrm{Mn}, \mathrm{P}, \mathrm{Mg}, \mathrm{Al}, \mathrm{Cr}, \mathrm{Sr}, \mathrm{Pb}, \mathrm{Ba}, \mathrm{Cd}, \mathrm{Co}, \mathrm{Mo}$ and $\mathrm{Ni}$ ) and carcass composition were subjected to one-way Analysis of Variance (Steel et al., 1996). The differences among treatments were compared by Tukey's Honesty Significant Difference Test and considered significant at $p<0.05$ (Snedecor and Cochran, 1991). The CoStat Computer Package (Version 6.303, PMB 320, Monterey, CA, 93940 USA) was used for statistical analysis.

\section{RESULTS}

Current results showed that there was a similar amount of minerals in all type of diets (Table III) but significant $(p<0.05)$ difference was recorded in mineral contents during analysis of faeces (Table IV). Overall lowest amount of minerals in $C$. catla fingerling faeces was observed when fed at $900 \mathrm{FTU} \mathrm{kg}^{-1}$ level followed by 600 FTU kg-1 level supplemented diet, significantly $(p<0.05)$ different from control and other phytase supplemented test diets. Whereas some of the minerals were noted the minimum in faeces at 600 and others were at 1200 FTU $\mathrm{kg}^{-1}$ level supplemented diet. However mineral discharge through faeces was recorded maximum when fish was fed with control diet ( $0 \mathrm{FTU} \mathrm{kg}^{-1}$ level). Results indicated that mineral discharge was decreased with increase in phytase addition and reached to its minimum at $900 \mathrm{FTU} \mathrm{kg}^{-1}$ level. It was noted that mineral excretion was increased with higher phytase supplementation upto $1500 \mathrm{FTU} \mathrm{kg}^{-1}$ level. Results showed that digestibility of $\mathrm{Ca}(76 \%), \mathrm{Na}(69 \%)$, $\mathrm{K}(76 \%), \mathrm{Fe}(70 \%), \mathrm{Cu}(70 \%), \mathrm{Zn}(75 \%), \mathrm{P}(73 \%), \mathrm{Mg}$ (75\%) and $\mathrm{Pb}(54 \%)$ were maximum at $900 \mathrm{FTU} \mathrm{kg}^{-1}$ level. Whereas, higher Mn (72\%) digestibility was observed in fish fed at $600 \mathrm{FTU} \mathrm{kg}^{-1}$ level based diet and it was statistically $(p<0.05)$ different from control and other test diets. On contrary, minerals such as $\mathrm{Al}(61 \%), \mathrm{Cr}(69 \%)$ as well as $\mathrm{Sr}(51 \%)$ were recorded highest at $1200 \mathrm{FTU} \mathrm{kg}^{-1}$ level and were statistically $(p<0.05)$ different from control and other test diets. However, other minerals such as $\mathrm{Ba}$, $\mathrm{Cd}, \mathrm{Co}, \mathrm{Mo}$ and $\mathrm{Ni}$ were not calculated as they were very

Table IV.- Analyzed mineral composition (\%) in faeces of $C$. catla fed phytase supplemented MOSM based test and control diets.

\begin{tabular}{lcccccc}
\hline Minerals & $\begin{array}{c}\text { Test diet }- \text { I } \\
\text { (Control diet) }\end{array}$ & Test diet - II & Test diet - III & Test diet - IV & Test diet - V & Test diet - VI \\
\hline Phytase levels & 0 & 300 & 600 & 900 & 1200 & 1500 \\
$\left(\right.$ FTU kg $\left.^{-1}\right)$ & & & & & & \\
$\mathrm{Ca}$ & $0.030 \pm 0.001 \mathrm{a}$ & $0.025 \pm 0.0004 \mathrm{~b}$ & $0.020 \pm 0.0004 \mathrm{c}$ & $0.017 \pm 0.001 \mathrm{~d}$ & $0.021 \pm 0.001 \mathrm{c}$ & $0.027 \pm 0.001 \mathrm{~b}$ \\
$\mathrm{Na}$ & $0.0038 \pm 0.001 \mathrm{a}$ & $0.0035 \pm 0.0005 \mathrm{ab}$ & $0.0028 \pm 0.0004 \mathrm{~cd}$ & $0.0025 \pm 0.001 \mathrm{~d}$ & $0.003 \pm 0.001 \mathrm{bc}$ & $0.0036 \pm 0.001 \mathrm{ab}$ \\
$\mathrm{K}$ & $0.77 \pm 0.04 \mathrm{a}$ & $0.67 \pm 0.02 \mathrm{~b}$ & $0.45 \pm 0.03 \mathrm{~d}$ & $0.34 \pm 0.02 \mathrm{e}$ & $0.57 \pm 0.03 \mathrm{c}$ & $0.68 \pm 0.03 \mathrm{~b}$ \\
$\mathrm{Fe}$ & $0.032 \pm 0.001 \mathrm{a}$ & $0.028 \pm 0.001 \mathrm{~b}$ & $0.024 \pm 0.001 \mathrm{c}$ & $0.018 \pm 0.0004 \mathrm{~d}$ & $0.022 \pm 0.001 \mathrm{c}$ & $0.029 \pm 0.002 \mathrm{ab}$ \\
$\mathrm{Cu}$ & $0.0019 \pm 0.0001 \mathrm{a}$ & $0.0017 \pm 0.0006 \mathrm{~b}$ & $0.0015 \pm 0.00004 \mathrm{c}$ & $0.0011 \pm 0.0001 \mathrm{e}$ & $0.0014 \pm 0.00004 \mathrm{~d}$ & $0.0017 \pm 0.0001 \mathrm{~b}$ \\
$\mathrm{Zn}$ & $0.022 \pm 0.0001 \mathrm{a}$ & $0.019 \pm 0.0005 \mathrm{~b}$ & $0.014 \pm 0.0003 \mathrm{~d}$ & $0.013 \pm 0.0003 \mathrm{~d}$ & $0.015 \pm 0.001 \mathrm{c}$ & $0.022 \pm 0.001 \mathrm{a}$ \\
$\mathrm{Mn}$ & $0.011 \pm 0.0004 \mathrm{a}$ & $0.01 \pm 0.0004 \mathrm{~b}$ & $0.0063 \pm 0.0002 \mathrm{~d}$ & $0.0076 \pm 0.0002 \mathrm{c}$ & $0.01 \pm 0.0002 \mathrm{~b}$ & $0.011 \pm 0.0003 \mathrm{a}$ \\
$\mathrm{P}$ & $1.26 \pm 0.04 \mathrm{a}$ & $1.08 \pm 0.03 \mathrm{~b}$ & $0.78 \pm 0.02 \mathrm{~d}$ & $0.61 \pm 0.02 \mathrm{e}$ & $0.91 \pm 0.01 \mathrm{c}$ & $1.13 \pm 0.04 \mathrm{~b}$ \\
$\mathrm{Mg}$ & $0.0061 \pm 0.0003 \mathrm{a}$ & $0.0052 \pm 0.0002 \mathrm{~b}$ & $0.0038 \pm 0.0003 \mathrm{c}$ & $0.0025 \pm 0.0002 \mathrm{~d}$ & $0.0032 \pm 0.0002 \mathrm{~cd}$ & $0.0050 \pm 0.0004 \mathrm{~b}$ \\
$\mathrm{Al}$ & $0.00036 \pm$ & $0.00031 \pm$ & $0.00028 \pm$ & $0.00024 \pm$ & $0.00022 \pm$ & $0.00027 \pm$ \\
& $0.00001^{\mathrm{a}}$ & $0.000003^{\mathrm{b}}$ & $0.00001^{\mathrm{c}}$ & $0.000003^{\mathrm{d}}$ & $0.00001^{\mathrm{e}}$ & $0.000002^{\mathrm{c}}$ \\
$\mathrm{Cr}$ & $0.020 \pm 0.002^{\mathrm{a}}$ & $0.015 \pm 0.001^{\mathrm{bc}}$ & $0.0094 \pm 0.001^{\mathrm{d}}$ & $0.011 \pm 0.002^{\mathrm{cd}}$ & $0.012 \pm 0.001^{\mathrm{bcd}}$ & $0.015 \pm 0.001^{\mathrm{ab}}$ \\
$\mathrm{Sr}$ & $0.00007 \pm$ & $0.000067 \pm$ & $0.000063 \pm$ & $0.000055 \pm$ & $0.000047 \pm$ & $0.000046 \pm$ \\
& $0.000002^{\mathrm{a}}$ & $0.000002^{\mathrm{ab}}$ & $0.000002^{\mathrm{b}}$ & $0.000002^{\mathrm{c}}$ & $0.000003^{\mathrm{d}}$ & $0.000003^{\mathrm{d}}$ \\
$\mathrm{Pb}$ & $0.0023 \pm 0.0002^{\mathrm{a}}$ & $0.0021 \pm 0.0001^{\mathrm{ab}}$ & $0.0019 \pm 0.0001^{\mathrm{bc}}$ & $0.0014 \pm 0.00003^{\mathrm{d}}$ & $0.0014 \pm 0.00004^{\mathrm{d}}$ & $0.0018 \pm 0.00002^{\mathrm{c}}$ \\
\hline
\end{tabular}

Means within rows having different superscripts are significantly different at $\mathrm{P}<0.05$. Data are means of three replicates. 
Table V.- Apparent mineral digestibility (\%) of $C$. catla fingerlings fed MOSM based test and control diets.

\begin{tabular}{lcccccc}
\hline Minerals & Test diet -I (control) & Test diet -II & Test diet -III & Test diet -IV & Test diet -V & Test diet -VI \\
\hline $\begin{array}{l}\text { Phytase levels } \\
\left(\mathrm{FTU} \mathrm{kg}^{-1}\right)\end{array}$ & 0 & 300 & 600 & 900 & 1200 & 1500 \\
$\mathrm{Ca}$ & $55.46 \pm 0.92^{\mathrm{f}}$ & $63.94 \pm 0.19^{\mathrm{d}}$ & $71.08 \pm 0.1^{\mathrm{b}}$ & $76.05 \pm 0.4^{\mathrm{a}}$ & $67.89 \pm 0.3^{\mathrm{c}}$ & $60.25 \pm 0.47^{\mathrm{e}}$ \\
$\mathrm{Na}$ & $51.22 \pm 0.67^{\mathrm{e}}$ & $56.29 \pm 0.47^{\mathrm{d}}$ & $65.76 \pm 0.54^{\mathrm{b}}$ & $69.05 \pm 0.74^{\mathrm{a}}$ & $61.19 \pm 0.9^{\mathrm{c}}$ & $54.71 \pm 0.77^{\mathrm{d}}$ \\
$\mathrm{K}$ & $45.62 \pm 0.87^{\mathrm{e}}$ & $53.08 \pm 0.63^{\mathrm{d}}$ & $68.94 \pm 0.84^{\mathrm{b}}$ & $76.50 \pm 0.95^{\mathrm{a}}$ & $59.02 \pm 0.53^{\mathrm{c}}$ & $52.47 \pm 0.65^{\mathrm{d}}$ \\
$\mathrm{Fe}$ & $43.10 \pm 0.05^{\mathrm{e}}$ & $49.18 \pm 0.59^{\mathrm{d}}$ & $59.73 \pm 0.92^{\mathrm{d}}$ & $69.57 \pm 0.36^{\mathrm{a}}$ & $62.24 \pm 0.73^{\mathrm{b}}$ & $50.08 \pm 0.68^{\mathrm{d}}$ \\
$\mathrm{Cu}$ & $46.58 \pm 0.45^{\mathrm{e}}$ & $50.41 \pm 0.28^{\mathrm{d}}$ & $56.88 \pm 0.61^{\mathrm{c}}$ & $70.33 \pm 0.93^{\mathrm{a}}$ & $59.59 \pm 1.25^{\mathrm{b}}$ & $51.57 \pm 0.54^{\mathrm{d}}$ \\
$\mathrm{Zn}$ & $54.90 \pm 0.7^{\mathrm{e}}$ & $61.68 \pm 0.31^{\mathrm{d}}$ & $73.23 \pm 0.22^{\mathrm{b}}$ & $75.51 \pm 0.53^{\mathrm{a}}$ & $68.64 \pm 0.57^{\mathrm{c}}$ & $56.23 \pm 0.8^{\mathrm{e}}$ \\
$\mathrm{Mn}$ & $48.11 \pm 0.71^{\mathrm{d}}$ & $56.20 \pm 0.46^{\mathrm{c}}$ & $71.93 \pm 0.22^{\mathrm{a}}$ & $66.73 \pm 0.46^{\mathrm{b}}$ & $55.3 \pm 0.95^{\mathrm{c}}$ & $48.39 \pm 0.85^{\mathrm{d}}$ \\
$\mathrm{P}$ & $43.76 \pm 0.72^{\mathrm{f}}$ & $51.81 \pm 0.78^{\mathrm{d}}$ & $65.65 \pm 0.87^{\mathrm{b}}$ & $73.28 \pm 0.54^{\mathrm{a}}$ & $58.67 \pm 0.37^{\mathrm{c}}$ & $49.70 \pm 0.21^{\mathrm{e}}$ \\
$\mathrm{Mg}$ & $35.22 \pm 0.56^{\mathrm{e}}$ & $45.40 \pm 0.58^{\mathrm{d}}$ & $60.22 \pm 0.67^{\mathrm{c}}$ & $74.75 \pm 0.94^{\mathrm{a}}$ & $65.94 \pm 0.74^{\mathrm{b}}$ & $47.26 \pm 0.7^{\mathrm{d}}$ \\
$\mathrm{Al}$ & $35.98 \pm 1.43^{\mathrm{e}}$ & $46.07 \pm 0.89^{\mathrm{d}}$ & $51.67 \pm 0.46^{\mathrm{c}}$ & $58.64 \pm 0.48^{\mathrm{b}}$ & $61.15 \pm 0.54^{\mathrm{a}}$ & $52.68 \pm 0.95^{\mathrm{c}}$ \\
$\mathrm{Cr}$ & $39.48 \pm 0.97^{\mathrm{d}}$ & $55.38 \pm 0.67^{\mathrm{c}}$ & $66.76 \pm 0.95^{\mathrm{b}}$ & $67.76 \pm 0.94^{\mathrm{ab}}$ & $69.37 \pm 0.99^{\mathrm{a}}$ & $53.92 \pm 0.93^{\mathrm{c}}$ \\
$\mathrm{Sr}$ & $26.12 \pm 1.06^{\mathrm{e}}$ & $31.06 \pm 0.47^{\mathrm{d}}$ & $37.39 \pm 0.93^{\mathrm{c}}$ & $43.68 \pm 0.78^{\mathrm{b}}$ & $50.98 \pm 0.85^{\mathrm{a}}$ & $52.84 \pm 0.98^{\mathrm{a}}$ \\
$\mathrm{Pb}$ & $23.83 \pm 0.99^{\mathrm{e}}$ & $29.02 \pm 0.98^{\mathrm{d}}$ & $37.51 \pm 0.63^{\mathrm{c}}$ & $54.49 \pm 0.79^{\mathrm{a}}$ & $52.28 \pm 0.82^{\mathrm{a}}$ & $40.70 \pm 0.96^{\mathrm{b}}$ \\
\hline
\end{tabular}

Means within rows having different superscripts are significantly different at $p<0.05$. Data are means of three replicates.

Table VI.- Proximate composition (\%) of $C$. catla carcass fed phytase supplemented MOSM based diet after 90-days feeding trial.

\begin{tabular}{lcccccc}
\hline Carcass parameters & Test diet -I (control) & Test diet -II & Test diet -III & Test diet -IV & Test diet -V & Test diet -VI \\
\hline $\begin{array}{l}\text { Phytase levels } \\
(\text { FTU kg })^{-1}\end{array}$ & 0 & 300 & 600 & 900 & 1200 & 1500 \\
Crude fat & $10.44 \pm 0.42^{\mathrm{d}}$ & $11.70 \pm 0.39^{\mathrm{c}}$ & $12.82 \pm 0.29^{\mathrm{b}}$ & $13.92 \pm 0.13^{\mathrm{a}}$ & $11.83 \pm 0.27^{\mathrm{c}}$ & $11.01 \pm 0.25^{\mathrm{cd}}$ \\
Crude protein & $55.47 \pm 0.45^{\mathrm{e}}$ & $57.67 \pm 0.38^{\mathrm{c}}$ & $60.59 \pm 0.36^{\mathrm{b}}$ & $62.35 \pm 0.19^{\mathrm{a}}$ & $58.40 \pm 0.25^{\mathrm{c}}$ & $56.44 \pm 0.35^{\mathrm{d}}$ \\
Carbohydrate & $17.49 \pm 1.16^{\mathrm{a}}$ & $16.12 \pm 0.68^{\mathrm{a}}$ & $13.89 \pm 0.67^{\mathrm{bc}}$ & $12.53 \pm 0.59^{\mathrm{c}}$ & $15.75 \pm 0.33^{\mathrm{ab}}$ & $16.67 \pm 0.49^{\mathrm{a}}$ \\
Ash & $8.41 \pm 0.35^{\mathrm{a}}$ & $7.27 \pm 0.44 \mathrm{~b}^{\mathrm{c}}$ & $6.61 \pm 0.36^{\mathrm{cd}}$ & $5.88 \pm 0.28^{\mathrm{d}}$ & $7.13 \pm 0.24^{\mathrm{bc}}$ & $7.99 \pm 0.13^{\mathrm{ab}}$ \\
Crude fiber & $1.25 \pm 0.02^{\mathrm{a}}$ & $1.18 \pm 0.05^{\mathrm{a}}$ & $1.07 \pm 0.06^{\mathrm{b}}$ & $0.93 \pm 0.03^{\mathrm{c}}$ & $1.17 \pm 0.04^{\mathrm{ab}}$ & $1.21 \pm 0.02^{\mathrm{a}}$ \\
Moisture & $6.94 \pm 0.17^{\mathrm{a}}$ & $6.06 \pm 0.28^{\mathrm{b}}$ & $5.01 \pm 0.13^{\mathrm{c}}$ & $4.39 \pm 0.34^{\mathrm{d}}$ & $5.71 \pm 0.14^{\mathrm{b}}$ & $6.68 \pm 0.09^{\mathrm{a}}$ \\
\hline
\end{tabular}

Means within rows having different superscripts are significantly different at $\mathrm{P}<0.05$. Data are means of three replicates.

low in diet (Table V). Lowest ADC\% of all the minerals observed for fish fed on control diet (0 FTU kg-1 level). Whereas ADC\% of minerals was decreased at the higher (1500 FTU kg-1) phytase supplementation in MOSM based diet.

Whole body analysis of fish fed phytase supplemented MOSM based diet is shown in Table VI. Least EE (10\%) and CP $(55 \%)$ retention in fish body was observed when fed with test diet-I ( 0 FTU kg-1 level). Whereas on the other hand maximum values of EE (14\%) and CP (62\%) were observed in fish fed on test diet-IV (900 FTU kg-1 level) based diet followed by fish that fed at test diet-III (600 FTU kg-1 level) i.e.13\% and 61\%, respectively. These values were significantly $(p<0.05)$ different from the fish fed on other phytase supplemented test diets as well as control diet (without phytase supplemented MOSM based diet). From these results it was found that improvement in fat and protein retention for $C$. catla fingerlings was started to improve at $300 \mathrm{FTU} \mathrm{kg}^{-1}$ level and reached to its maximum when fingerlings were fed with 900 FTU $\mathrm{kg}^{-1}$ supplemented MOSM based test diet, whereas further increase in phytase supplementation (1200 and $1500 \mathrm{FTU}$ $\mathrm{kg}^{-1}$ level) could not enhance nutrient retention in fish body. Minimum amount of carbohydrates (13\%) and crude fiber (1\%) contents were analyzed in fish fed at $900 \mathrm{FTU} \mathrm{kg}^{-1}$ level supplemented diet as compared to other test diets and control diet (0 FTU kg-1 level). However maximum values of carbohydrate $(17 \%)$ and crude fiber $(1 \%)$ were observed 
in fish fed at 0 FTU $\mathrm{kg}^{-1}$ level based diet (control diet) that was statistically $(p<0.05)$ similar with the fish that fed at 300 FTU kg $^{-1}$ level supplemented diet. Similarly minimum moisture (4\%) and ash (6\%) contents were recorded in fish fed at $900 \mathrm{FTU} \mathrm{kg}^{-1}$ level supplemented based diet that was significantly $(p<0.05)$ different from the fish which fed phytase supplemented and non-supplemented MOSM based diets (control diet). However maximum moisture $(7 \%)$ and ash $(8 \%)$ contents were found in the fish fed at $0 \mathrm{FTUkg}^{-1}$ level supplemented diet (control) that was statistically $(p<0.05)$ similar with the fish fed on 1500 FTUkg-1 level supplemented MOSM based diets. These findings indicate that a decline in carbohydrates, crude fiber, moisture and ash contents was observed when fish fed on phytase supplemented test diets at 300 FTUkg $^{-1}$ level. Lowest values for these parameters were noted in the fish fed at 900 FTU $\mathrm{kg}^{-1}$ level supplemented based diet and started to increase with the increase in phytase supplementation in fish feed up to $1500 \mathrm{FTU} \mathrm{kg}^{-1}$ level.

These findings showed that phytase supplementation is compulsory for the higher mineral digestibility and nutrient $(\mathrm{CP}$ and $\mathrm{EE})$ retention in fish body resulting in improved fish performance. Improvement in mineral digestibility and carcass composition was started from 300 FTU kg-1 level and reached to the maximum at 900 FTU $\mathrm{kg}^{-1}$ level after which it gradually decreased after the further increase in phytase supplementation upto $1500 \mathrm{~kg}^{-1}$ level. It was found that $900 \mathrm{FTU} \mathrm{kg} \mathrm{kg}^{-1}$ level of phytase supplementation is the most suitable level for the higher $\mathrm{ADC} \%$ of minerals and maximum deposition of protein and lipids in fish body that are important for fish performance.

\section{DISCUSSION}

Phytate commonly exists in plant based ingredients that usually binds with divalent cations and is known as a major anti-nutritional factor. Phytate makes minerals unavailable to fish and decrease their digestibility due to the binding between phytate complex and major minerals (Oh et al., 2004; Nwanna et al., 2007). Breakdown of complex chelated structure of phytate enhances the release and utilization of essential minerals. Researchers indicated that phytate present in plant by-products may chelate with some of the important minerals i.e. Fe, $\mathrm{Ca}, \mathrm{Mn}, \mathrm{Cu}, \mathrm{Ni}, \mathrm{Cr}$, $\mathrm{Na}, \mathrm{K}, \mathrm{P}$ and Mg (Cao et al., 2007; Dersjant-Li et al., 2015; Hussain et al., 2015a, b). From present study, it was noted that $900 \mathrm{FTU} \mathrm{kg}^{-1}$ is the most optimum level of phytase supplementation that can increase mineral digestibility for C. catla fingerlings fed MOSM based diet. Whereas, some of the minerals showed maximum digestibility for fingerlings when they were fed with $600 \mathrm{FTU} \mathrm{kg}^{-1}$ level and remaining were fed at $1200 \mathrm{FTU} \mathrm{kg}^{-1}$ level supplemented diet. It was found that phytase influences the mineral digestibility from 250 to $1500 \mathrm{FTU} \mathrm{kg}^{-1}$ levels in different fish species at different environmental conditions (Cao et al., 2007). Increased mineral utilization was also observed by Cheng and Hardy (2002), when they supplemented plant meal based diets with phytase that liberated chelated minerals from phytate present in plant feed stuffs. Similar to our findings, Hussain et al. (2015b) noted that anti nutritional factors in soybean meal based diets such as phytate played negative role in mineral digestibility, whereas phytase supplementation at $1000 \mathrm{FTU} \mathrm{kg}^{-1}$ level improved mineral digestibility by breaking down the chelated phytateminerals complex resulting in maximum utilization of essential minerals by fish and decreased mineral discharge in water. Almost similar with present results, Van-Weerd et al. (1999) concluded that phytase addition at $1000 \mathrm{FTU}$ $\mathrm{kg}^{-1}$ level in soybean meal based diet showed maximum $\mathrm{ADC} \%$ of $\mathrm{P}$ in Clarias gariepinus. Our results are also supported by the findings of Hussain et al. (2015a). They reported significantly $(p<0.05)$ higher digestibility values of minerals for L. rohita fingerlings fed cottonseed meal based diet supplemented with 750 and $1000 \mathrm{FTU} \mathrm{kg}^{-1}$ level. While, Hussain et al. (2016) found that $750 \mathrm{FTU} \mathrm{kg}^{-1}$ is the optimum level for maximizing $\mathrm{ADC} \%$ of minerals in $L$. rohita fingerlings. On the other hand, maximum $\mathrm{ADC} \%$ of $\mathrm{P}$ had been claimed in tra cat fish juveniles fed soybean meal based diet supplemented at higher level of phytase e.g. $1500 \mathrm{FTU} \mathrm{kg}^{-1}$ (Hung et al., 2015). Variations in optimal level of phytase supplementation may be due to difference in plant ingredients used in diet formulation and experimental fish species (Baruah et al., 2007a). In contrary to current results, Baruah et al. (2007b) found maximum mineral digestibility values in Labeo rohita fingerlings fed at $500 \mathrm{FTU} \mathrm{kg}^{-1}$ level in plant meal based diets. They concluded that minerals such as $\mathrm{Fe}, \mathrm{Mg}, \mathrm{K}, \mathrm{Mn}, \mathrm{P}$ and $\mathrm{Na}$ showed highest $\mathrm{ADC} \%$ at $500 \mathrm{FTU} \mathrm{kg}^{-1}$ level as compared to control and other phytase supplemented test diets. In contrary to these findings and present results, Laining et al. (2010) observed highest mineral digestibility and absorption in Takifu gurubripes (tiger puffer), when fed at the level of $2000 \mathrm{FTU} \mathrm{kg}^{-1}$ in soybean meal based diet. In another study Nwanna and Olusola (2014) suggested that phytase supplementation played a non-significant role in term of mineral digestibility in Oreochromis niloticus, Nile tilapia fingerlings. They found little improvement in mineral digestibility at a very high dose $\left(8000 \mathrm{FTU} \mathrm{kg}^{-1}\right)$ of phytase supplementation. Their results were not in specific range of phytase supplementation (250 to $1500 \mathrm{FTU} \mathrm{kg}^{-1}$ level) as narrated by Cao et al. (2007). Hussain et al. (2016) reported maximum minerals utilization by L. rohita fingerlings fed at $400 \mathrm{FTU} \mathrm{kg}^{-1}$ level and 4\% citric acid 
supplemented cottonseed meal based test diet. However, it was noted that this difference in phytase supplementation levels may be dependent on the amount of phytate in plant meal based diet, $\mathrm{pH}$ of digestive system, fish species, experimental conditions feed processing methods, feed drying methods, quality and type of phytase and methods used for feed preparation (Baruah et al., 2007a; DersjantLi et al., 2015). According to these studies and current results, higher mineral digestibility and utilization in fingerlings resulted in minimum water pollution and maximum performance of fish after the use of phytase in fish feed.

It was found that phytate is a chelated complex compound that usually binds with important nutrients such as $\mathrm{CP}, \mathrm{EE}$ and other nutrients making them unavailable to fish, resulting in poor fish carcass composition (Cao et al., 2007). Maximum retention of CP (62\%) and EE (14\%) were recorded in C. catla fingerlings fed at 900 FTU kg ${ }^{-1}$ level as compared to control and remaining phytase supplemented test diets. However, lowest values of $\mathrm{CP} \%$ and $\mathrm{EE} \%$ in fish were observed at $0 \mathrm{FTU} \mathrm{kg}^{-1}$ level (control diet). Similarly, different researchers also found that phytase supplementation improves the protein retention in fish body when fed on plant by-products based diets (Storebakken et al., 1998; Khajepour et al., 2012). Increasing nutrient bioavailability would positively affect body composition and bone strength when fish fed on phytase supplemented soybean meal based diet (Sardar et al., 2007). Very close findings to our results were reported by Cheng et al. (2015). They found maximum protein retention in yellow catfish (Pelteobagrus fulvidraco), when fed phytase supplemented plant meal based diet supplemented with $1000 \mathrm{FTU} \mathrm{kg}^{-1}$. Whereas, in contradiction, microbial phytase supplementation at 500 FTU kg-1 level in the diet of Pangasius pangasius increased the apparent protein retention in fish body (Debnath et al., 2005). Soybean meal based phytase supplemented diets enhanced nutrient retention in $C$. gariepinus at levels ranging from $750 \mathrm{FTU} \mathrm{kg}^{-1}$ and 1000 FTU $\mathrm{kg}^{-1}$ levels and the values obtained at these levels were significantly different from the values obtained from the diet without phytase supplementation (Akpoilih et al., 2016). In current study $900 \mathrm{FTU} \mathrm{kg}^{-1}$ level was found as the best level for increasing nutrient retention in fish body, that was in optimal ranges (250-1500 FTU kg-1) reported by Cao et al. (2007), whereas Olusola and Nwanna (2014) found a very high level of phytase dose (8000 FTU kg-1 level) for improvement in protein retention in O. niloticus fingerlings fed processed soybean meal based diets. Nearly similar results were observed by Yoo and Bai (2014). They found maximum crude fat in Paralichthys olivaceus (Olive Flounder) when fed phytase supplemented soybean meal based diet supplemented at $1000 \mathrm{FTU} \mathrm{kg}^{-1}$ level. Highest body lipid contents were recorded when tiger puffer fed phytase supplemented plant protein of soybean, corn and wheat meal based diet. This divergence in enzyme doses may be due to ingredient composition, the presence or absence of the stomach, fish species as well as types of phytase (Baruah et al., 2007a).

Lowest body crude fiber (1\%), ash (6\%) and moisture (4\%) contents were observed in the $C$. catla fingerlings when they fed at 900 FTU $\mathrm{kg}^{-1}$ level supplemented MOSM based diets. Similar to present results, increase in CP contents, resulted in decreased moisture and crude ash values were noted in the red sea bream fed phytase supplemented plant meal based diet (Hossain et al., 2007). Moisture contents were also recorded lower in catfish when fed phytase supplemented plant protein based diet as compared to control diet (Hung et al., 2015). Similar to our results, Vielma et al. (2004) found minimum fish carcass ash and also significantly reduced nutrient contents in fish when fed with phytase deficient diets. Phytase supplemented plant protein based diets of Ictalurus punctatus (channel catfish) showed highest ash contents as compared to control diet (Jackson et al., 1996). Pham et al. (2008) also observed an increase in ash contents of Paralichthys olivaceus (Olive Flounder) fed phytase supplemented cottonseed meal and soybean meal based diets. On contrary, Cheng et al. (2015) found no difference in moisture contents of whole fish body fed with or without phytase supplemented diets. In contrast, higher contents of moisture were recorded in C. carpio when fed at 0 FTU $\mathrm{kg}^{-1}$ level supplemented based diet (Sardar et al., 2007).

\section{CONCLUSION}

Current research work provided sufficient evidences about phytase supplementation that breakdown the phytate complex present in MOSM based diets. From these findings, it was concluded that supplementation of phytase at $900 \mathrm{FTU} \mathrm{kg}^{-1}$ level played a major role in improving $\mathrm{ADC} \%$ of minerals and carcass composition of $C$. catla fingerlings as compared to control diet (without phytase supplementation). Addition of phytase also decreases the cost of fish feed by improving the quality of plant meal based diet as compared to costly fish meal.

Statement of conflict of interest

Authors have declared no conflict of interest.

\section{REFERENCES}

Abdulkadir, A.R., Zawawi, D.D. and Jahan, M.S., 2016. Proximate and phytochemical screening 
of different parts of Moringa oleifera. Russian Agric. Sci., 42: 34-36. https://doi.org/10.3103/ S106836741601002X

Abdulkarim, S.M., Long, K., Lai, O.M., Muhammad, S.K.S. and Ghazali, H., 2005. Some physicochemical properties of Moringa oleifera seed oil extracted using solvent and aqueous enzymatic methods. J. Fd. Chem., 93: 253-263. https://doi. org/10.1016/j.foodchem.2004.09.023

Akpoilih, U.B., Emmanuel, K.A. and Bamidele, O.O., 2016. Dietary phytase improves growth and water quality parameters for juvenile Clarias gariepinus fed soyabean diet-based diets. Int. J. Aquacul., 5: $1-20$.

Allan, G.L. and Rowland, S.J., 1992. Development of an experimental diet for silver perch (Bidynus bidyanus). In: Austasia aquaculture magazine, $6^{\text {th }}$ ed. AustAsia Aquaculture, East Victoria Park, W.A., pp. 39-40.

Anjorin, T.S., Ikokoh, P. and Okolo, S., 2010. Mineral composition of Moringa oleifera leaves, pods and seeds from two regions in Abuja, Nigeria. Int. J. Agric. Biol., 12: 431-434.

AOAC, 2000. Official methods of analysis, $19^{\text {th }}$ edn. Association of Official Analytical Chemists, Arlington, VA.

Aslam, S., Abbas, S., Kalhoro, M.A. and Shoaib, A., 2016. Anchor worms (lernaeid parasites), Lernaea polymorpha yü and Lernaea cyprinacea (copépode: lernaeidae) on major carps at different fish farms in Punjab, Pakistan. Sci. Int., 28: 295-298.

Baruah, K., Pal, K. A. K., Narottam, P.S. and Debnath, D., 2007a. Microbial phytase supplementation in rohu, Labeo rohita, diets enhances growth performance and nutrient digestibility. J. World Aquacul. Soc., 38: 129-137. https://doi.org/10.1111/ j.1749-7345.2006.00081.x

Baruah, K., Sahu, P.N., Pal, K.A., Jain, K.K., Debnath, D. and Mukherjee, C.S., 2007b. Dietary microbial phytase and citric acid synergistically enhances nutrient digestibility and growth performance of Labeo rohita (Hamilton) juveniles at sub-optimal protein level. Aquacul. Res., 38: 109-120. https:// doi.org/10.1111/j.1365-2109.2006.01624.x

Cao, L., Wang, W., Yang, C., Yang, Y., Diana, J., Yakupitiyage, A., Luo, Z. and Li, D., 2007. Application of microbial phytase in fish feed. $J$. Enzyme Microb. Tech., 40: 497-507. https://doi. org/10.1016/j.enzmictec.2007.01.007

Chabi, I.B., Kayodé, A.P.P., Agassoussi, O.A.S., Agbobatinkpo, P.B., Chikou, A. and Codjia, J.T.C., 2015. Development and bioefficacy study of plant- based proteins diets for juvenile African catfish. $J$. appl. Biosci., 94: 8801-8808.

Cheng, Z.J. and Hardy, R.W., 2002. Effect of microbial phytase on apparent nutrient digestibility of barley, canola meal, wheat and wheat middlings, measured in vivo using rainbow trout (Oncorhynchus mykiss). Aquacul. Nutr., 8: 271-277. https://doi. org/10.1046/j.1365-2095.2002.00219.x

Cheng, N., Chen, P., Lei, W., Feng, M. and Wang, C., 2015. The sparing effect of phytase in plant-proteinbased diets with decreasing supplementation of dietary $\mathrm{NaH}_{2} \mathrm{PO}_{4}$ for juvenile yellow catfish Pelteobagrus fulvidraco. Aquacul. Res., 47: 39523963. https://doi.org/10.1111/are.12845

Christopher, M.A., Caipang, R., Dechavez, B. and Amar, A.J.M., 2011. Potential application of microbial phytase in aquaculture, ELBA Bioflux, 3: 1.

Chu, Z.J., Yu, D.H., Dong, G.F. and Gong, S.Y., 2015. Partial replacement of fish meal by soybean meal with or without methionine and phytase supplement in the diets for juvenile Chinese sucker. Aquacul. Nutr., 22: 989-996. https://doi.org/10.1111/ anu. 12318

Dawood, M.A.O., Koshio, S., Ishikawa, M. and Yokoyama, S., 2015. Effects of partial substitution of fish meal by soybean meal with or without heatkilled Lactobacillus plantarum (LP20) on growth performance, digestibility, and immune response of Amberjack, Seriola dumerili juveniles. BioMed Res. Int., 2015: Article ID 514196. https://doi. org/10.1155/2015/514196

Debnath, D., Pal, A.K., Sahu, N.P., Jain, K.K., Yengkokpam, S. and Mukherjee, S.C., 2005. Effects of dietry microbial phytase supplementation on growth and nutrient digestibility of Pangasius pangasius fingerlings. J. Aquacul. Res., 36: 180-187. https://doi.org/10.1111/j.1365-2109.2004.01203.x

Dersjant-Li, Y., Awati, A., Schulze, H. and Partridge, G., 2015. Phytase in non-ruminant animal nutrition: A critical review on phytase activities in the gastrointestinal tract and influencing factors. J. Sci. Fd. Agric., 95: 878-896. https://doi.org/10.1002/ jsfa.6998

Enterria A., Slocum, M., David, A., Panayotis, B., Karayannakidis, D. and Lee, C.M., 2011. Partial replacement of fish meal with plant protein sources singly and in combination in diets for summer flounder (Paralichthys dentatus). World Aquacult. Soc., 42: 253-265. https://doi.org/10.1111/j.17497345.2011.00533.x

Essa,A.M., Mabrouk,A.H. and Zaki,A.M., 2004. Growth performance of grass carp, Ctenopharyngodon 
idella and hybrid grass carp fingerlings fed on different types of aquatic plants and artificial diet in concrete basins. Egyptian J. Aquacul. Res., 30B: 341-348. https://doi.org/10.1590/S141552732008000400007

FAO, 2014. Psetta maxima (Linnaeus, 1758). Statistical information, global aquaculture production 19502012. Fisheries and Aquaculture Department, FAO, Rome, Italy.

FAO, 2015. Cultured aquatic species information programme, Catla catla (Hamilton, 1822). Fisheries and Aquaculture Department, FAO, Rome, Italy. http://www.fao.org/fishery/culturedspecies/Catla catla/en

Ferreira, P.M.P., Farias, D.F., Oliveira, J.T.A. and Carvalho, A.F.U., 2008. Moringa oleifera: Bioactive compounds and nutritional potential. Rev. Nutr., 21: 431-437.

Hossain, M.A., Pandey, A. and Satoh, S., 2007. Effects of organic acids on growth and phosphorus utilization in red sea bream Pagrus major. Fish. Sci., 73: 1309-1317.

Hung, S.W., Tu, C.Y. and Wang, W.S., 2007. In vitro effects of singular or combined anti-oxidative vitamins and/or minerals on tilapia (Oreochromis hybrids) peripheral blood monocyte-derived, anterior kidney-derived, and spleen-derived macrophages. Fish Shellf. Immunol., 23: 1-15. https://doi.org/10.1016/j.fsi.2006.08.020

Hung, L.T., Thanh, N.T., Pham, M.A. and Browdy, C.L., 2015. A comparison of the effect of dietary fungal phytase and dicalcium phosphate supplementation on growth performances, feed and phosphorus utilization of tra catfish juveniles (Pangasianodon hypophthalmus). Aquacul. Nutr., 21: 10-17. https:// doi.org/10.1111/anu.12134

Hussain, S.M., Afzal, M., Rana, S.A., Javed, A. and Iqbal, M., 2011. Effect of phytase supplementation on growth performance and nutrient digestibility of Labeo rohita fingerlings fed on corn gluten mealbased diets. Int. J. Agric. Biol., 13: 916-922.

Hussain, S. M., Hameed, T., Afzal, M., Mubarik, M. S., Asrar, M., Shah, S. Z. H., Ahmad, S., Arsalan, M. Z. H., Riaz, D., Tahir, N., Amber, F., Shahzad, M. M. and Khichi, T. A. A., 2014. Effects of phytase supplementation on mineral digestibility in Cirrhinus mrigala fingerlings fed on sunflower meal-based diets. Int. J. Biosci., 5: 173-181. https:// doi.org/10.12692/ijb/5.12.173-181

Hussain, S.M., Shahzad, M.M., Afzal, M., Javid, A., Mubarik, M.S., Shah, S.Z.H., Hussain, M., Ahmad, S., Arsalan, M.Z.H., Manzoor, R. and
Riaz, D., 2015a. Efficacy of phytase enzyme for increasing mineral digestibility of Cirrhinus mrigala fingerlings fed on soybean meal-based diet. Pakistan J. Zool., 47: 1807-1816.

Hussain, S.M., Afzal, M., Javid, A., Hussain, A.I., Ali, Q., Mustafa, I., Chatha S.A.S., Shah S.Z.H., Hussain, M. and Ullah, M.I., 2015b. Efficacy of phytase supplementation on growth performance and mineral digestibility of Labeo rohita fingerlings fed on cottonseed meal based diet. Pakistan J. Zool., 47: 699-709.

Hussain, S.M., Ahmad, S., Shahzad, M.M., Arsalan, M.Z.H., Riaz, D., Ahmad, N., Tabassum, S. and Ahmed, A.W., 2016. Mineral digestibility of Labeo rohita fingerlings fed on cottonseed meal based diets supplemented with citric acid and phytase enzyme. Int. J. Biosci., 8: 25-35. https://doi. org $/ 10.12692 / \mathrm{ijb} / 8.2 .25-35$

Hussain, S.M., Hameed, T., Afzal, M., Javid, A., Aslam, N., Shah, S.Z.H., Hussain, M. and Shahzad, M.M., 2017. Growth performance and nutrient digestibility of Cirrhinus mrigala fingerlings fed phytase supplemented sunflower meal based diet. Pakistan J. Zool., 49:1713-1724. http://dx.doi. org/10.17582/journal.pjz/2017

Jackson, L., Li, M.H. and Robinson, E.H., 1996. Use of microbial phytase in channel catfish Ictalurus punctatus diets to improve utilization of phytate phosphorus. J. World Aquacul. Soc., 27: 309313. https://doi.org/10.1111/j.1749-7345.1996. tb00613.x

Jondreville, C., Hayler, R. and Feuerstein, D., 2005. Replacement of zinc sulphate by microbial phytase for piglets given a maize-soya-bean meal diet. $J$. Anim. Sci., 81: 77-83. https://doi.org/10.1079/ ASC41440077

Khajepour, F., Hosseini, S.A. and Imanpour, M.R., 2012. Dietary crude protein, citric acid and microbial phytase and their interacts to influence growth performance, muscle proximate composition and hematocrit of common carp, Cyprinus carpio L., juveniles. World J. Zool., 7: 118-122.

Laining, A., Ishikawa, M., Kyaw, K., Gao, J., Binh, N.T., Koshio, S., Yamaguchi, S., Yokoyama, S. and Koyama, J., 2011. Dietary calcium/phosphorus ratio influences the efficacy of microbial phytase on growth, mineral digestibility and vertebral mineralization in juvenile tiger puffer, Takifugu rubripes. Aquacul. Nutr., 17: 267-277. https://doi. org/10.1111/j.1365-2095.2009.00749.x

Lei, X.G., Weaver, J.D., Mullaney, E., Ullah, A.H. and Azain, M.J., 2013. Phytase a new life for 
an old enzyme. Annu. Rev. Anim. Biosci., 1: 283-309. https://doi.org/10.1146/annurevanimal-031412-103717

Liener, I.E., 1994. Implications of antinutritional components in soybean foods. Crit. Rev. Fd. Sci. Nutr., 34: 31-67. https://doi. org/10.1080/10408399409527649

Lim, S.J. and Lee, K.J., 2009. Partial replacement of fish meal by cottonseed meal and soybean meal with iron and phytase supplementation for parrot fish Oplegnathus fasciatus. Aquaculture, 290: 283-289. https://doi.org/10.1016/j.aquaculture.2009.02.018

Liu, H., Jin, J., Zhu, X., Han, D., Yang, Y. and Xie, S., 2015. Effect of substitution of dietary fish meal by soybean meal on different sizes of gibel carp (Carassius auratus gibelio): Digestive enzyme gene expressions and activities, and intestinal and hepatic histology. Aquacul. Nutr., 23: 129-147. https://doi.org/10.1111/anu.12375

Liu, L.W., Su, J.M., Zhang, T., Liang, X.Z. and Luo, Y.L., 2013. Apparent digestibility of nutrients in grass carp diet supplemented with graded levels of phytase using pre-treatment and spraying methods. Aquacul. Nutr., 19: 91-99. https://doi.org/10.1111/ j.1365-2095.2012.00942.x

Lovell, R.T., 1989. Nutrition and feeding of fish. Van Nostrand-Reinhold, New York, pp. 260. https://doi. org/10.1007/978-1-4757-1174-5

Makkar, H.P.S. and Becker, K., 2009. Jatropha curcas, a promising crop for the generation of biodiesel and value added coproduct. Eur. J. Lipid Sci. Tech., 111: 773-787. https://doi.org/10.1002/ ejlt.200800244

Muin, H., Taufek, N.M., Abiodunr, A., Yusof, H.M. and Razak, S.A., 2015. Effect of partial and complete replacement of fishmeal with mushroom stalk meal and soy bean meal on growth performance of Nile tilapia, Oreochromis niloticus fingerlings. Sains Malay., 44: 511-516. https://doi.org/10.17576/jsm2015-4404-05

NRC, 1993. Nutrient requirements of fish. National Research Council, National Academy Press, Washington, DC.

Noureddini, H. and Dang, J., 2009. Degradation of phytate in distillers' grains and corn gluten feed by Aspergillus niger phytase. Appl. Biochem. Biotechnol., 159: 11-23. https://doi.org/10.1007/ s12010-008-8365-2

Nwanna, L.C. and Olusola, B.S., 2014. Effect of supplemental phytase on phosphorus digestibility and mineral composition in Nile tilapia (Oreochromis niloticus). Int. J. Aquacul., 4: 89-95. https://doi.org/10.5376/ija.2014.04.0015

Nwanna, L.C., Eisenreich, R. and Schwarz, F.J., 2007. Effect of wet-incubation of dietary plant feedstuffs with phytases on growth and mineral digestibility by common carp Cyprinus carpio L. Aquaculture, 271: $\quad 461-468$. https://doi.org/10.1016/j. aquaculture.2007.04.020

Oh, B.C., Choi, W.C., Park, S., Kim, Y.O. and Oh, T.K., 2004. Biochemical properties and substrate specificities of alkaline and histidine acid phytases. Appl. Microbiol. Biotechnol., 63: 362-372. https:// doi.org/10.1007/s00253-003-1345-0

Olusola, S.E. and Nwanna, L.C., 2014. Growth performance of Nile tilapia (Oreochromis niloticus) fed processed soybean meal based diets supplemented with phytase. Int. J. Aquacul., 4: 4854. https://doi.org/10.5376/ija.2014.04.0008

Pham, M.A., Lee, K.J., Dang, T.M., Lim, S.J., Ko, G.Y., Eo, J. and Oh, D.H., 2008. Improved apparent digestibility coefficient of protein and phosphorus by supplementation of microbial phytase in diets containing cotton seed and soybean meal for juvenile olive flounder (Paralichthys olivaceus). $J$. Anim. Sci., 21: 1367-1375.

Plaipetch, P. and Yakupitiyage, A., 2014. Effect of replacing soybean meal with yeast-fermented canola meal on growth and nutrient retention of Nile tilapia, Oreochromis niloticus (Linnaeus 1758). Aquacul. Res., 45: 1744-1753.

Rana, K.J., Siriwardena, S. and Hasan, M.R., 2009. Impact of rising feed ingredient prices on aquafeeds and aquaculture production. Food and Agriculture Organization of the United Nations, Paper. No. 541, FAO, Rome, Italy, pp. 63.

Robinson, E.H., Li, M.H. and Manning, B.B., 2002. Comparison of microbial phytase and dicalciumphosphate or growth and bone mineralization of pond raised channel catfish, Ictalurus punctatus. J. appl. Aquacul., 12: 81-88.

Rowland, S.J. and Ingram, B.A., 1991. Diseases of Australian native fishes. In: Fisheries bulletin 4. NSW Fisheries, Sydney, NSW, Australia.

Salem, H.B. and Makkar, H.P.S., 2009. Defatted Moringa oleifera seed meal as a feed additive for sheep. Anim. Feed Sci. Tech., 150: 27-33. https:// doi.org/10.1016/j.anifeedsci.2008.07.007

Sardar, P., Randhawa, H.S., Abid, M. and Prabhakar, S.K., 2007. Effect of dietry microbial phytase supplementation on growth performance, nutrient utilization, body compositions and haematobiochemical profiles of Cyprinus carpio (L.) fingerlings fed soyprotein-based diet. Aquacul. 
Nutr., 13: 444-456. https://doi.org/10.1111/j.13652095.2007.00497.x

Snedecor, G.W. and Cochran, W.G., 1991. Statistical methods, $8^{\text {th }}$ Ed. Iowa State University Press, Ames., USA, pp. 503.

Steel, R.G.D., Torrie, J.H. and Dickey, D.A., 1996. Principles and procedures of statistics, $3^{\text {rd }} \mathrm{Ed}$. McGraw Hill International Book Company Inc., New York, USA, pp. 336-352.

Storebakken, T., Shearer K.D. and Roem, A.J., 1998. Availability of protein, phosphorus and other elements in fishmeal, soy-protein concentrate and phytase-treated soy-protein concentrate-based diets to Atlantic salmon, Salmo salar. Aquaculture, 161: 365-379. https://doi.org/10.1016/S00448486(97)00284-6

Tacon, A. and Metian, M., 2013. Fish matters: Importance of aquatic foods in human nutrition and global food supply. Rev. Fish. Sci., 21: 22-38. https://doi.org/10.1080/10641262.2012.753405

Van-Weerd, J.H., Khalaf, K.H.A. Aartsen, F.J. and Tijssen, P.A.T., 1999. Balance trials with African cat fish, Clarias gariepinus fed phytase-treated soybean meal-based diets. Aquacul. Nutr., 5: 135-142. https://doi.org/10.1046/j.1365-2095.1999.00100.x
Vielma, J., Makinen, T., Ekholm, P. and Koskela, J., 2000. Checked the influence of dietary soy and phytase levels on performance and body composition of large rainbow trout, Oncorhynchus mykiss and algal availability of phosphorus load. Aquaculture, 183: 349-362. https://doi.org/10.1016/ S0044-8486(99)00299-9

Vielma, J., Ruohonen, K., Gabaudan, J. and Vogel, K., 2004. Top-spraying soybean meal-based diets with phytase improves protein and mineral digestibilities but not lysine utilization in rainbow trout, Oncorhynchus mykiss (Walbaum). Aquacul. Res., 35: 955-964. https://doi.org/10.1111/j.13652109.2004.01106.x

Weiss, EA., 1971. Castor, sesame, and safflower. Leonard Hill, London.

Yao, B. and Fan, Y.L., 2000. Molecular biology and gene engineering of phytase. Chinese J. Biotech., 16: $1-5$.

Yoo, G. and Bai, S.C., 2014. Effects of the dietary microbial phytase supplementation on bioavailability of phosphorus in juvenile olive flounder Paralichthys olivaceus fed soybean meal based diets. Fish. aquat. Sci., 17: 319-324. https:// doi.org/10.5657/FAS.2014.0319 